\title{
Relationship between serum concentrations of leptin, soluble leptin receptor, testosterone and IGF-I, and growth during the first year of postnatal life in the male rhesus monkey, Macaca mulatta
}

\author{
Ganapathy K Bhat, Tony M Plant ${ }^{1}$ and David R Mann \\ Department of Physiology and the Cooperative Reproductive Science Research Center, Morehouse School of Medicine, Atlanta, Georgia 30310, \\ USA and ${ }^{1}$ Department of Cell Biology and Physiology and the Specialized Cooperative Center Program of Reproductive Research, University of Pittsburgh \\ School of Medicine, Pittsburgh, Pennsylvania 15261, USA
}

(Correspondence should be addressed to D R Mann, Cooperative Reproductive Science Research Center, Morehouse School of Medicine, 720 Westview Drive SW, Atlanta, Georgia 30310, USA; Email: mann@msm.edu)

\begin{abstract}
Objectives: Subnormal leptin levels in low birth weight infants may allow for catch-up growth during infancy. Scant data are available that relate growth with circulating leptin during normal infancy in primates. The current study objective was to examine the association between serum leptin, its soluble receptor (sOB-R), testosterone and IGF-I concentrations, and body weight during infancy in male rhesus monkeys.

Design: Hormone levels were assessed longitudinally in animals $(n=7)$ from birth until 1 year of age. Results: Body weight increased during the first 6 months of life and was strongly correlated with rising IGF-I levels and, as IGF-I plateaued and then declined during the second half of the year, body weight gain decelerated. In contrast, leptin levels declined gradually with age during the first year of life in conjunction with increasing body weight. There was no association between body weight gain and serum leptin levels or between serum testosterone and leptin values. Since sOB-R levels also declined with leptin values, it does not appear that levels of bioavailable leptin changed during infancy. Conclusions: The data do not support the contention that leptin regulates growth during infancy, but the close association between IGF-I levels and body weight suggested that this hormone may regulate growth in infant male monkeys. The failure to observe an association between serum testosterone and leptin concentrations suggested that leptin is not involved in the activation of the hypothalamic-pituitary-testicular axis during this developmental period.
\end{abstract}

European Journal of Endocrinology 153 153-158

\section{Introduction}

In humans, growth during the first 2 years of life is particularly dramatic with growth velocity exceeding that at any other stage of postnatal development, and body weight increasing by as much as two- to threefold (1). The hormonal regulation of growth during this phase of development involves several major endocrine systems, in particular the thyroid, adrenal and growth hormone $(\mathrm{GH})$ axes (1).

More recently, leptin has also been implicated in this process. In this regard, circulating leptin appears to be positively correlated with growth during fetal development $(2-7)$, as reflected in a positive association between cord blood leptin and birth weight $(3,7)$. Postnatally, however, the relationship between circulating leptin levels and growth remains unclear. In one study, blood leptin levels were inversely correlated with body weight gain over the first 4 months of postnatal life, a relationship that remained evident at 24 months of age (3). In contrast, other investigators (7) found that from birth until 2 years of age, there was no correlation between serum leptin and body weight in either normal birth weight infants or infants with intrauterine growth retardation.

One of the reasons why this issue has not been explored in any greater detail is that it would require repeated assessment of developmental changes in circulating leptin concentrations in normal infants. The rhesus monkey is an excellent paradigm of human development and reproduction $(8,9)$ and should be useful as a surrogate for the human infant for such a study. In terms of a developmental time-frame, the first year of postnatal life in the Old World monkey 
may be viewed as analogous to the first 3-4 years of human life (10).

The objective of this study was to explore longitudinally the relationship between developmental changes in circulating levels of leptin, its soluble receptor (sOB-R), insulin-like growth factor-I (IGF-I) and testosterone and growth (body weight gain) during the first year of postnatal life in the male monkey. sOB-R and IGF-I levels were determined because the former affects the bioavailability of leptin (11-13), and the latter is of potential importance as a determinant of postnatal growth (14).

\section{Materials and methods}

\section{Animals}

Seven male rhesus monkeys that were produced by the Breeding Program of the Center for Research in Reproductive Physiology at the University of Pittsburgh were employed for this study. The animals were born within a span of 2 years between 1997 and 2000. Birth weights of these animals ranged from 478 to $578 \mathrm{~g}$ (means S.E.M.; $520 \pm 12 \mathrm{~g}$ ). Initially, each infant was housed with its mother in a single cage. At 8-11 months of age (9.4 \pm 0.5 months) the infants were separated from their mothers and for the remainder of the study were housed with one or more animals of comparable age.

\section{Experimental protocol}

With six of the monkeys, blood samples were drawn by femoral venipuncture under sedation (ketamine hydrochloride; $10-30 \mathrm{mg} / \mathrm{kg}$ body weight, i.m.) between 0830 and $1030 \mathrm{~h}$ at biweekly intervals beginning at 1 or 3 weeks of age. In the seventh animal, blood sampling was started at 11 weeks of age. Body weight was determined on the day of venipuncture. Serum samples were stored at $-20{ }^{\circ} \mathrm{C}$ until assayed. This study was conducted according to the principles and procedures of the National Institutes of Health Guidelines for the Care and Use of Laboratory Animals and approved by the Institutional Animal Care and Use Committee of the University of Pittsburgh.

\section{Assays}

Serum concentrations of leptin and testosterone were determined in singulate by radioimmunoassay using commercially available kits (Linco Research, Inc., St Charles, MO, USA and Diagnostic Products Corporation, Los Angeles, CA, USA respectively). The leptin assay was previously validated by one of our laboratories for the rhesus monkey (15). The minimum detection limits for the leptin and testosterone assay were $0.24 \mathrm{ng} / \mathrm{ml}$ and $0.65 \mathrm{ng} / \mathrm{ml}$ respectively. Serum concentrations of IGF-I were assayed using a commercially available human non-extraction, two-site immunoradiometric assay kit
(Diagnostic Systems Laboratories, Webster, TX, USA) that was validated in our laboratory for the rhesus monkey. The minimum detection limit for the IGF-I assay was $2.1 \mathrm{ng} / \mathrm{ml}$. sOB-R was measured using an enzyme-linked immunosorbent kit for human sOB-R (Diagnostic Systems Laboratories). In this assay, aliquots of serum samples from male and female rhesus monkeys ran parallel to the human standard. The minimum detection limit for this assay was $0.2 \mathrm{ng} / \mathrm{ml}$. The intraassay coefficients of variation for the leptin, testosterone, IGF-I and sOB-R assays were respectively 11.7, 6.3, 4.0 and $2.1 \%$; the interassay coefficients of variation were $16.5,18.8,4.1$ and $6.2 \%$.

\section{Statistics}

Body weight and serum concentrations of leptin, IGF-I, testosterone and sOB-R are expressed as means \pm S.E.M. Hormonal and body weight data were analyzed by oneway ANOVA with repeated measures over time (age). In addition, linear regression analysis (Pearson's correlation test, two-tailed) was performed to assess the relationship between age, leptin, sOB-R, IGF-I, testosterone, body weight and growth rate. We also employed partial correlation analyses (JMP-IN; SAS Institute, Cary, $\mathrm{NC}$, USA) to control for the influence of other related variables on the linear relationship between a pair of variables (e.g. if variables $\mathrm{R}$ and $\mathrm{S}$ are correlated with one another, how is that correlation affected by variables $\mathrm{U}, \mathrm{V}$ and $\mathrm{W}$ ?). The partial correlation assesses the 'true' strength of a relationship between two variables by taking into account the effect that other related variables have on that relationship. Growth rate (velocity) for individual animals was calculated as the increase in body weight per week over the first year of life. Body weight data were also partitioned into the first and second half of the first year of life and regression analysis was performed to test ( $t$-test) the hypothesis of the equality of the slopes (i.e. to determine whether body weight gain differed between these two developmental periods).

\section{Results}

Mean body weight in the seven infants increased $(P<0.0001)$ linearly and more than threefold over the first 6 months of postnatal life (Fig. 1, top). Weight velocity then decreased during the second half of the first year of life (slope for first half $=40.5 \mathrm{~g} /$ week versus $28.0 \mathrm{~g} /$ week for the second half; the hypothesis of the equality of the two slopes was rejected at a level of $P=0.0012$ ). Growth rate during the first postnatal year was unrelated to birth weight $(r=-0.090$, $P=0.848$ ) in these animals (data not shown).

Serum leptin and sOB-R concentrations, which exhibited marked variation within and between animals, were highest shortly after birth and declined gradually over the next 12 months (Fig. 1, bottom). 


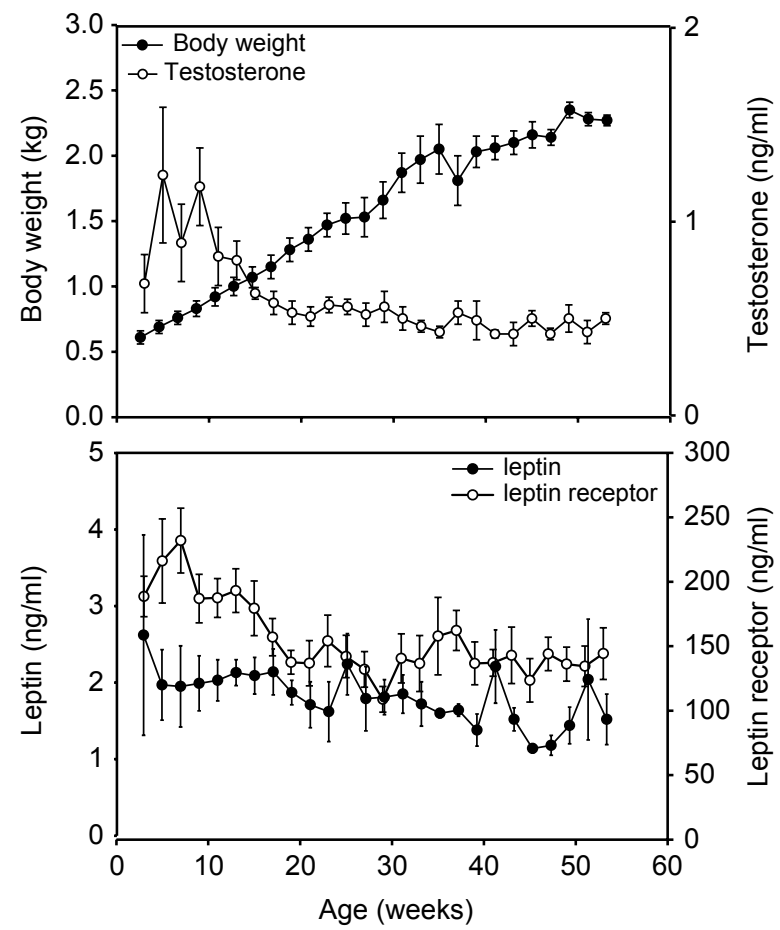

Figure 1 Mean \pm S.E.M. (top) body weight and serum testosterone concentrations and (bottom) serum leptin and SOB-R concen-

trations in male rhesus monkey over the first year of postnatal life.

The age-related changes in leptin did not reach levels of significance (by one-way ANOVA), but there was a significant negative association between leptin and age (Fig. 2, top; $r=-0.660, P<0.0001$ ) and leptin and body weight (Fig. 2, bottom; $r=-0.664$, $P<0.0001)$. Mean serum sOB-R concentrations declined significantly with age $(P<0.0001$ by ANOVA), and developmental changes in sOB-R were positively correlated with changes in its ligand $(r=0.447, P=0.022)$.

Partial correlation analyses indicated that there was no significant association between serum leptin levels and body weight during postnatal weeks 1 through $27(r=-0.0354, P=0.743)$ or weeks 29 through $53(r=-0.188, P=0.109)$. In addition, growth velocity was unrelated to serum leptin concentrations over these same periods $(r=0.0703, P=0.518$ and $r=-0.0316, P=0.794$ respectively).

Serum testosterone concentrations changed significantly with age $(P<0.0001$; Fig. 1 , top $)$. Levels of this steroid increased after birth to reach a peak of approximately $1.5 \mathrm{ng} / \mathrm{ml}$ at $5-10$ weeks of age before declining progressively to reach basal values of approximately $0.8 \mathrm{ng} / \mathrm{ml}$ at 20 weeks of age. There was no significant association between serum leptin and testosterone concentrations during the first year of life (partial correlation for full year $=-0.0155, P=0.849$ ).

Serum IGF-I levels increased more than tenfold from birth until 27 weeks of age (by repeated measure
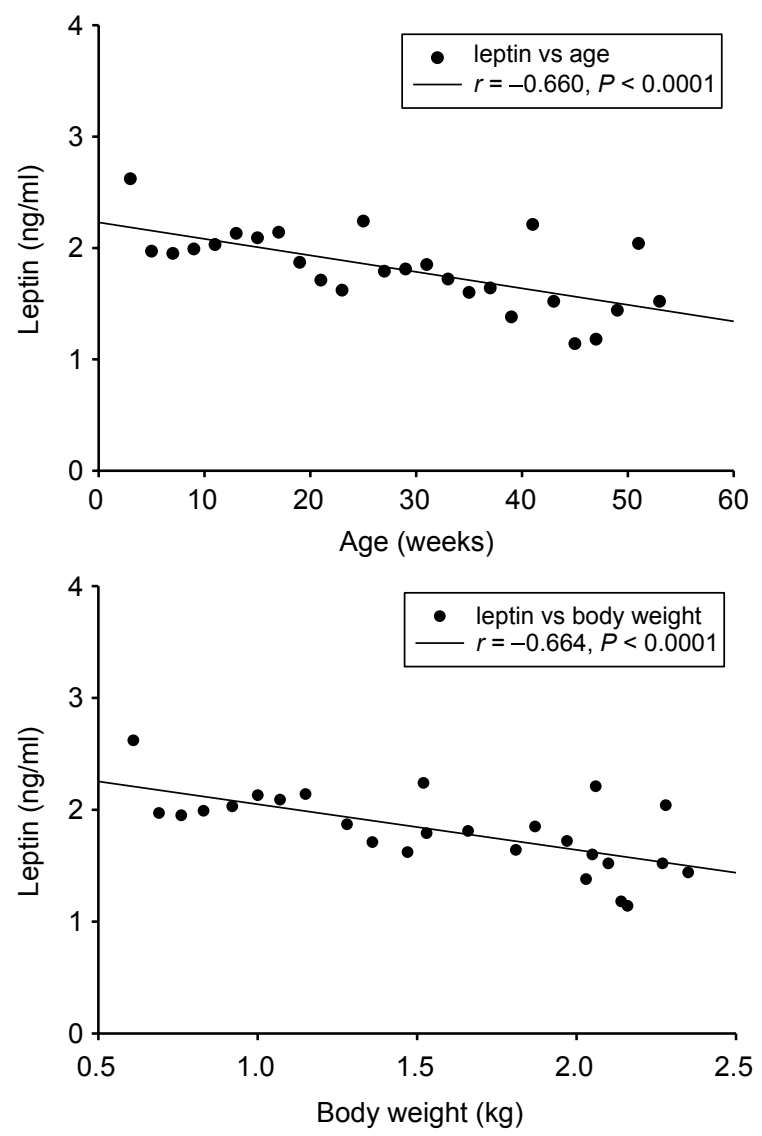

Figure 2 Correlations between mean serum leptin concentrations and (top) age and (bottom) body weight in male rhesus monkey over the first year of postnatal life.

ANOVA, $P<0.0001$ ), and then gradually declined (by repeated measure ANOVA, $P=0.014$ ) during the second half of the first year of postnatal life (Fig. 3, top). During the period from birth to 27 weeks of age, there was a highly significant positive association between serum IGF-I and body weight $(r=0.446$, $P<0.001$ ) (Fig. 3, bottom). This relationship remained significant $(r=0.468, P=0.0001)$ when controlled for other variables (age, leptin, leptin receptor and testosterone; Fig. 4, top). Linear regression analyses indicated that for weeks 1 through 27 there was no significant relationship between mean serum IGF-I and leptin, but there was a significant inverse relationship between serum IGF-I and the sOB-R $(r=-0.856$, $P<0.0001)$. When controlled for the other variables (partial correlations), however, this association between serum IGF-I values and serum sOB-R levels was no longer significant.

During the second half of the year when weight gain decelerated and IGF-I levels declined, the relationship between IGF-I and body weight was reversed and the two variables showed negative association $(r=-0.275, P=0.019)$. This association of IGF-I and body weight persisted when the other variables 

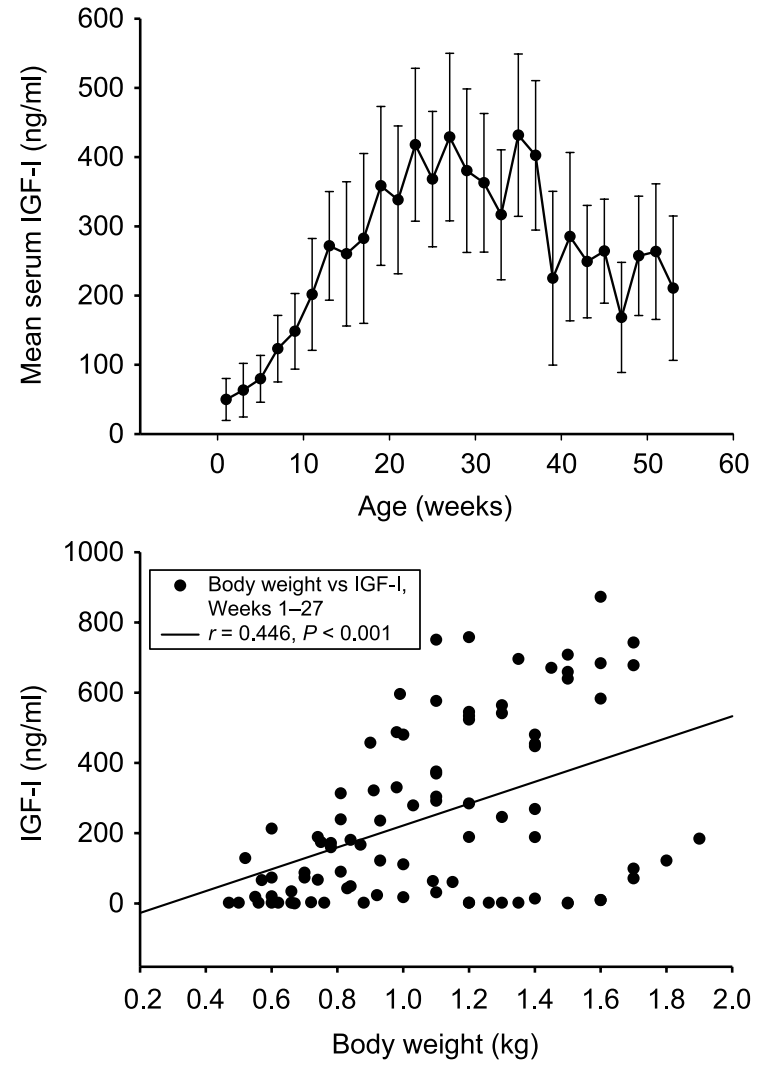

Figure 3 (Top) Mean \pm S.E.M. serum IGF-I concentrations in male rhesus monkey over the first year of postnatal life. (Bottom) Correlation of serum IGF-I concentrations and body weight in individual monkeys for postnatal weeks 1-27.

were controlled (partial correlation, $r=-0.302$, $P=0.012$; Fig. 4 , bottom). Despite the close association between serum IGF-I and body weight during the first year of life, weekly change in body weight was not associated with serum IGF-I levels in these animals.

\section{Discussion}

In the present study, there was a highly significant positive association between serum IGF-I levels and body weight during the first 6 postnatal months in male monkeys. IGF-I levels were lowest shortly after birth and increased in conjunction with increasing body weight over the first half year of postnatal life but, during the second half of the year, body weight gain decelerated in association with a significant decrease in serum IGF-I levels. Despite the close association between serum IGF-I and body weight, we were unable to detect any significant independent association between weekly change in body weight and serum IGF-I levels in these animals.

It has previously been reported that IGF-I levels were positively predictive of body weight gain in
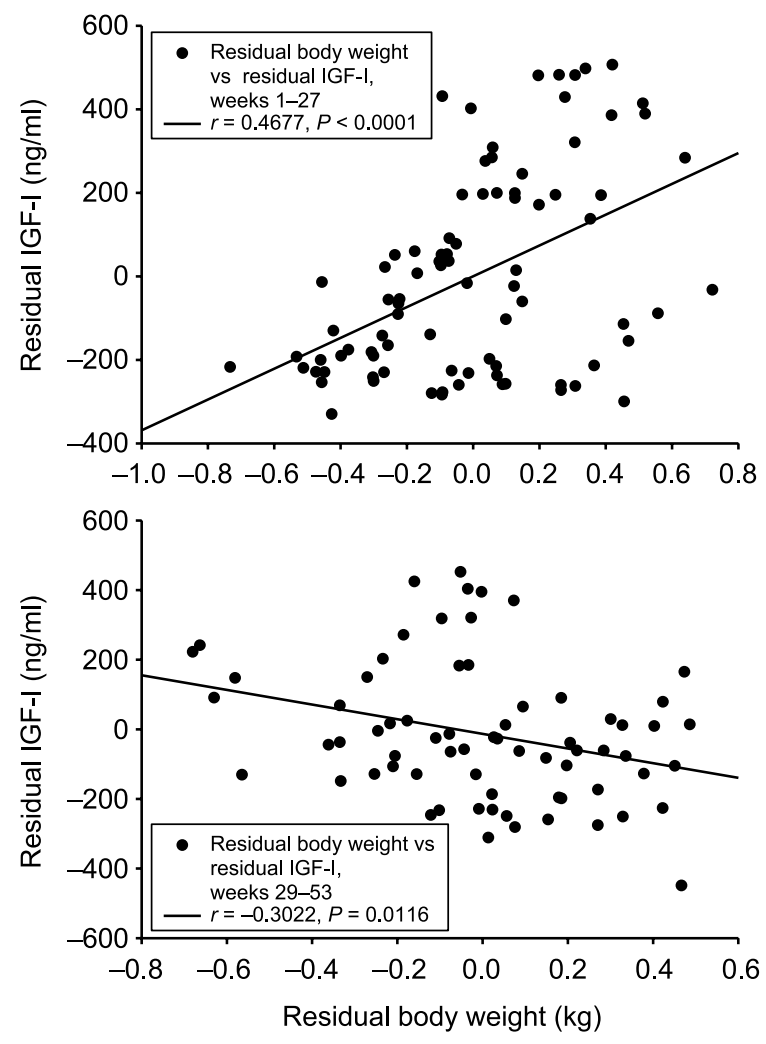

Figure 4 Partial correlations of serum IGF-I and body weight during (top) postnatal weeks 1-27 and (bottom) weeks 29-53.

infant monkeys that were treated with recombinant human GH for the first 7 weeks of postnatal life or in those infants whose mothers were treated with the hormone during pregnancy and/or lactation (16). These data suggest that IGF-I is involved in regulating growth during infancy in primates, and are consistent with previous results in humans indicating that IGF-I plays a prominent role in catch-up growth during infancy in intrauterine growth-retarded infants (17). In these infants, IGF-I levels were reduced at 5 days of age, but increased to control values by 12 months of age in those subjects that had complete catch-up growth during their first year of life.

Another interesting finding of the current study was the marked change in the developmental pattern of circulating IGF-I concentrations that occurred between the first and second half of the first year (i.e. rapid rise and then a decline) of postnatal life in male monkeys. This change appeared to be tightly linked to the time when infants were weaned and separated from their mothers. Whether this association is causal is not known although it is recognized that nutritional intake is a primary regulator of IGF-I concentrations during the early postnatal period in primates and rodents $(16,18)$. It is possible that the decline in IGF-I levels is related to a shift to all solid foods when the animals were separated from their mothers. 
Both leptin and sOB-R concentrations declined gradually in concert with age and, as a result, the ratio of leptin to sOB-R in the circulation did not change significantly over the first year of postnatal life in male monkeys. Thus, it appears unlikely that age-related changes in the sOB-R affected the bioavailability of circulating leptin. Cross-sectional studies of boys and girls have provided information on mean leptin levels from 0 to 3 years of age $(12,13)$, but longitudinal data for this period of human development have not been reported. This is the first study to provide longitudinal data relating developmental changes in circulating leptin with growth over this period in a higher primate.

In the present study, there was a negative association between leptin and body weight throughout the first year of life. Moreover, growth velocity was unassociated with mean leptin values during this period. Partial correlations performed to ascertain whether other variables (age, testosterone, IGF-I and sOB-R) might be masking the effect of leptin on body weight and growth velocity failed to detect any significant association between these growth parameters and serum leptin concentrations. Our results, therefore, support an earlier human study describing an absence of a correlation between serum leptin and body weight in either normal term infants or those with intrauterine growth retardation (7). Thus, leptin may not play a substantial role in the regulation of growth in higher primates during infancy.

It is possible, as others have suggested (7), that infancy and early childhood represent a period of relative insensitivity of the central nervous system to the anorexigenic effects of leptin. This would appear to be advantageous to the growing primate infant, allowing body weight and body fat mass increases without the negative impact of leptin on energy intake and expenditure. In fact, body mass index (BMI) increased over the first year of life in both normal infants and those with intrauterine growth retardation, and BMI was positively associated with serum leptin concentrations, but only in normal infants (7). While we have no direct evidence from the present study that the sensitivity to leptin is reduced during infancy, variability in leptin within and between monkeys was relatively high in the present study and is perhaps reflective of a system regulating energy balance that has not fully matured.

Parenthetically, it should also be noted that birth weight in the current study, and therefore presumably cord blood leptin, was unrelated to body weight gain during the first year of postnatal life. Thus these data do not support the idea that lower circulating leptin levels during 'normal' infancy in primates is associated with a greater rate of growth as has been suggested in studies with growth-retarded human infants (3).

Infancy in the male primate is characterized by increased activity within the hypothalamic-pituitarygonadal (HPG) axis (8). In the present study and as reported earlier $(19,20)$, daytime testosterone concentrations decreased after an initial peak at 5-9 weeks of age to low basal values by 4 months of postnatal life and remained low throughout the rest of the first year. Since leptin plays an important, albeit permissive, role in the pubertal resurgence of gonadotropin-releasing hormone release in primates (21), we were interested in any relationship between circulating leptin and activation of the HPG axis as reflected by developmental changes in circulating testosterone. The failure to observe a significant relationship between serum testosterone and leptin concentrations during infancy in male monkeys suggests that leptin does not play a role in the activation of the HPG axis during this period of development.

In conclusion, the data from the current longitudinal study are the first to detail developmental relationships between circulating concentrations of leptin, sOB-R, IGF-I and testosterone and growth during normal infancy in higher primates. We found that there was a strong positive association between circulating IGF-I levels and the increase in body weight over the first year of life, suggesting that developmental changes in IGF-I may be serving as the major regulatory factor for growth in the infant male primate. In contrast, the results do not support the hypothesis that circulating leptin regulates growth during infancy in these animals. The large level of variability in serum leptin concentrations between and within individual monkeys over this developmental period is perhaps suggestive of a leptin regulatory system that is not fully matured at this time. The present study also failed to provide evidence for a role of leptin in regulating the HPG axis of the infant primate.

\section{Acknowledgements}

We would like to thank Dr Lewis VanBrackle (Department of Mathematics, Kennesaw State University, Kennesaw, GA, USA) for his help with the statistical analysis of the present data. The authors also acknowledge the expert technical assistance of Michael A Cicco, Robert Beidler and Carolyn Phalin and the support of the Primate and Assay Cores of the Specialized Cooperative Centers Program in Reproduction Research at the University of Pittsburgh School of Medicine.

\section{Funding}

This work was supported by NIH grants HD41749, RR03024, and GM08248 to Morehouse School of Medicine and HD13254 and HD08610 to the University of Pittsburgh School of Medicine.

\section{References}

1 Reiter EO \& Rosenfeld RG. Normal and aberrant growth. In Williams Textbook of Endocrinology, edn. 10, pp 1003-1104. Eds PR Larsen, HM Kronenberg, S Melmed \& KS Polonsky. Philadelphia: Saunders, 2003. 
2 Matsuda J, Yokota I, Iida M, Murakami T, Naito E, Ito M, Shima K \& Kuroda Y. Serum leptin concentration in cord blood: relationship to birth weight and gender. Journal of Clinical Endocrinology and Metabolism $1997 \mathbf{8 2} 1642-1644$.

3 Ong KK, Ahmed ML, Sherriff A, Woods KA, Watts A, Golding J \& Dunger DB. Cord blood leptin is associated with size at birth and predicts infancy weight gain in humans. Journal of Clinical Endocrinology and Metabolism 199984 1145-1148.

4 Ertl T, Funke S, Sarkany I, Szabo I, Rascher W, Blum WF \& Sulyok E. Postnatal changes of leptin levels in full-term and preterm neonates: their relation to intrauterine growth, gender and testosterone. Biology of the Neonate $199975167-176$.

5 Schulz S, Hackel C \& Weise W. Hormonal regulation of neonatal weight: placental leptin and leptin receptors. British Journal of Obstetrics and Gynaecology 2000107 1486-1491.

6 Harigaya A, Onigata K, Nako Y, Nagashima K \& Morikawa A. Role of serum leptin in the regulation of weight gain in early infancy. Biology of the Neonate 199975 234-238.

7 Jaquet D, Leger J, Tabone MD, Czernichow P \& Levy-Marchal C. High serum leptin concentrations during catch-up growth of children born with intrauterine growth retardation. Journal of Clinical Endocrinology and Metabolism 199984 1949-1953.

8 Plant TM. Puberty in primates. In The Physiology of Reproduction, pp 453-485. Eds E Knobil \& JD Neill. New York: Raven Press, 1994.

9 Grumbach MM. The neuroendocrinology of human puberty revisited. Hormone Research 200257 (Suppl 2) 2-14.

10 Bogin B. Growth and development: recent evolutionary and biocultural research. In Biological Anthropology: The State of the Science, pp 49-70. Eds NT Boaz \& LD Wolfe. Bend: International Institute for Evolutionary Research, 1995.

11 Quinton ND, Smith RF, Clayton PE, Gill MS, Shalet S, Justice SK, Simon SA, Walters S, Postel-Vinay MC, Blakemore AIF \& Ross RJM. Leptin binding activity changes with age: the link between leptin and puberty. Journal of Clinical Endocrinology and Metabolism 199984 2336-2341.

12 Mann DR, Johnson AOK, Gimpel T \& Castracane D. Changes in circulating leptin, leptin receptor, and gonadal hormones from infancy until advanced age in humans. Journal of Clinical Endocrinology and Metabolism 200388 3339-3345.

13 Kratzsch J, Lammert A, Bottner A, Seidel B, Mueller G, Thiery J, Hebebrand J \& Kiess W. Circulating soluble leptin receptor and free leptin index during childhood, puberty and adolescence. Journal of Clinical Endocrinology and Metabolism 200287 4587-4594.

14 Rosen CJ \& Pollak M. Circulating IGF-1: new perspectives for a new century. Trends in Endocrinology and Metabolism 199910 $136-141$.

15 Plant TM \& Durrant AR. Circulating leptin does not appear to provide a signal for triggering the initiation puberty in the male rhesus monkey. Endocrinology 1997138 4505-4508.

16 Wilson ME, Gordon TP, Chikazawa K, Gust D, Tanner JM \& Rudman CG. Effects of growth hormone on neonatal growth in nursing rhesus monkeys. Journal of Clinical Endocrinology and Metabolism 199172 1302-1307.

17 Thieriot-Prevost G, Boccara JF, Francoual C, Badoual J \& Job JC. Serum insulin-like growth factor 1 and serum growth-promoting activity during the first postnatal year in infants with intrauterine growth retardation. Pediatric Research 198824 380-383.

18 Phillips A, Drakenberg K, Persson B, Sjogren B, Eklof AC, Hall K \& Sara V. The effects of altered nutritional status upon insulingrowth factors and their binding proteins in neonatal rats. Pediatric Research 198926 128-134.

19 Plant TM. A striking diurnal variation in plasma testosterone concentrations in infantile male rhesus monkeys (Macaca mulatta). Neuroendocrinology 198235 370-373.

20 Mann DR, Davis-DaSilva M, Wallen K, Coan P, Evans DE \& Collins DC. Blockade of neonatal activation of the pituitary-testicular axis with continuous administration of a gonadotropinreleasing hormone agonist in male rhesus monkeys. Journal of Clinical Endocrinology and Metabolism 198459 207-211.

21 Mann DR \& Plant TM. Leptin and pubertal development. Seminars in Reproductive Medicine 200220 93-102.

Received 14 January 2005

Accepted 29 March 2005 